\title{
Influence of cross-diffusion on the fecally-orally epidemic model with spatial heterogeneity
}

\author{
Jing Ge ${ }^{1,2}$, Zhigui Lin²*i( ) and Qunying Zhang ${ }^{2}$
}

"Correspondence:

zglin68@hotmail.com

${ }^{2}$ School of Mathematical Science,

Yangzhou University, Yangzhou, 225002, China

Full list of author information is available at the end of the article

\begin{abstract}
A strongly coupled cooperative parabolic system, which describes fecally-orally epidemic model with cross-diffusion in a heterogeneous environment, was formulated and analyzed. The basic reproduction number $R_{0}^{D}$, which serves as a threshold parameter that predicts whether the coexistence will exist or not, is introduced by the next infection operator and the related eigenvalue problems. By applying upper and lower solutions method, we present the sufficient conditions for the existence of the coexistence solution. The true positive solutions can also be obtained by monotone iterative method. Our results imply that the fecally-orally epidemic model with cross-diffusion admits at least one coexistence solution when the basic reproduction number exceeds one and the cross-diffusion coefficient is sufficiently small, while no coexistence exists when the basic reproduction number is smaller than one or the cross-diffusion coefficient is large enough. Finally, some numerical simulations are exhibited to confirm our analytical findings.
\end{abstract}

MSC: Primary 35J47; secondary 92D30

Keywords: fecally-orally epidemic model; eigenvalue problem; basic reproduction number; cross-diffusion

\section{Introduction}

Since the most influential and theoretical model, the SIR model, was formulated by Kermack and McKendrick in 1927, the geographic transmission of infectious diseases has been becoming an important issue in mathematical epidemiology. Capasso and PaveriFontana [1] proposed a spatially independent system to investigate the cholera epidemic which spread in the European Mediterranean regions in 1973. The epidemic model involves a positive feedback interaction between the infected human population and the concentration of bacteria. Considering the impact of spatial diffusion, Capasso and Maddalena [2] discussed the reaction-diffusion system

$$
\begin{cases}u_{t}-d_{1} \Delta u=-a_{11} u+a_{12} v, & t>0, x \in \Omega, \\ v_{t}-d_{2} \Delta v=-a_{22} v+g(u), & t>0, x \in \Omega,\end{cases}
$$

(c) The Author(s) 2017. This article is distributed under the terms of the Creative Commons Attribution 4.0 International License (http://creativecommons.org/licenses/by/4.0/), which permits unrestricted use, distribution, and reproduction in any medium, provided you give appropriate credit to the original author(s) and the source, provide a link to the Creative Commons license, and indicate if changes were made. 
subject to three different boundary conditions to interpret fecally-orally transmitted epidemics such as typhoid fever, infections hepatitis, polyometitis etc. Here $d_{1}, d_{2}, a_{11}, a_{12}$ and $a_{22}$ are the positive constants, $u(x, t)$ and $v(x, t)$, respectively, represent the spatial densities of the bacterial population and of the human population infected by the bacteria at location $x$ in the habitat $\Omega$ and at time $t \geq 0, \frac{1}{a_{11}}$ is the mean lifetime of the agent in the environment, $\frac{1}{a_{22}}$ denotes the mean infectious period of the human infections, $\frac{1}{a_{12}}$ is the multiplicative factor of the mean infectious agent due to the human populations, and $g(u)$ represents the infection rate of human under the assumption that total susceptible human population is constant during the evolution of the epidemic.

To understand the dynamics of solutions to problem (1.1) and its corresponding Cauchy problem, traveling waves solutions were studied in [3, 4], and the entire solutions (that is, solutions defined for all times $t \in \mathbb{R}$ and for all points $x \in \mathbb{R}$ ) was established in [5, 6] and the spreading fronts of an infective environment was given in [7] by considering a free boundary problem.

In the past few years, a great deal of mathematical models have been developed to investigate the impact of diffusion and spatial heterogeneity on the dynamics of diseases $[8$, 9]. Allen et al. [8] proposed a frequency-dependent SIS (susceptible-infected-susceptible) epidemiological reaction-diffusion system, which reads as follows:

$$
\begin{cases}S_{t}-d_{S} \Delta S=-\frac{\beta(x) S I}{S+I}+\gamma(x) I, & x \in \Omega, t>0, \\ I_{t}-d_{I} \Delta I=\frac{\beta(x) S I}{S+I}-\gamma(x) I, & x \in \Omega, t>0, \\ \frac{\partial S}{\partial \eta}=\frac{\partial I}{\partial \eta}=0, & x \in \partial \Omega, t>0, \\ S(x, 0)=S_{0}(x) \geq 0, \quad I(x, 0) \geq I_{0}(x) \geq, \not \equiv 0, & x \in \bar{\Omega},\end{cases}
$$

the authors of [8] studied the existence, uniqueness, stability of the disease-free equilibrium and particularly the asymptotical behavior of the unique endemic equilibrium as the dispersal rate of susceptible $d_{S}$ tends to zero.

On the other hand, in ecology, different concentration levels of species can affect the diffusive direction of another interacting species, which is called cross-diffusion [10]. This is also another hot issue and attracts much attention in recent years; see [11-16] and the references therein. Combined the spatial heterogeneity and cross-diffusion, the corresponding ecosystem can induce more complicated dynamical behaviors $[17,18]$.

In present paper, based on the model in [2], we will focus on the following cross-diffusion epidemic model in a spatially heterogeneous environment with Dirichlet boundary condition:

$$
\begin{cases}u_{t}-\Delta\left[\left(d_{1}+\alpha_{1} u+\frac{\beta_{1}}{\gamma_{1}+\nu}\right) u\right]=a_{12}(x) v-a_{11}(x) u, & x \in \Omega, t>0, \\ v_{t}-\Delta\left[\left(d_{2}+\frac{\beta_{2}}{\gamma_{2}+u}+\alpha_{2} v\right) v\right]=g(u)-a_{22}(x) v, & x \in \Omega, t>0, \\ u(x, 0)=v(x, 0)=0, & x \in \partial \Omega,\end{cases}
$$

where $\Omega$ is a bounded domain in $\mathbb{R}^{N}(N \geq 1)$ with smooth boundary $\partial \Omega, a_{11}(x), a_{12}(x)$ and $a_{22}(x)$ defined on $\bar{\Omega}$ are all sufficiently smooth and strictly positive functions. $\frac{1}{a_{11}(x)}$ is the mean lifetime of the agent at location $x, \frac{1}{a_{22}(x)}$ denotes the mean infectious period of the human infections and $\frac{1}{a_{12}(x)}$ is the multiplicative factor of the mean infectious agent at location $x$. The constants $d_{i}, \alpha_{i}, \beta_{i}$ and $\gamma_{i}$ are nonnegative $(i=1,2)$. The term $d_{1}+\alpha_{1} u+\frac{\beta_{1}}{\gamma_{1}+v}$ 
represents the 'self-diffusion' and $\frac{\beta_{1}}{\gamma_{1}+\nu} u$ implies that the chaseable capacity of the bacteria $u$ is decreasing with the enhanced resistance of the infected human $v[19,20]$.

We further assume that $g: \mathbb{R} \rightarrow \mathbb{R}$ satisfies the following hypothesis:

(H1) $g \in C^{1}([0, \infty)), g(0)=0, g^{\prime}(z)>0, \forall z \geq 0$;

(H2) $\frac{g(z)}{z}$ is decreasing and $\limsup _{z \rightarrow \infty} \frac{g(z)}{z}<\min _{\bar{\Omega}}\left\{\frac{a_{11}(x)}{a_{12}(x)}\right\} \cdot \min _{\bar{\Omega}} a_{22}(x)$.

From the view of ecology, we are more interested in investigating the steady state of problem (1.3), namely, the corresponding strongly coupled elliptic problem

$$
\begin{cases}-\Delta\left[\left(d_{1}+\alpha_{1} u+\frac{\beta_{1}}{\gamma_{1}+\nu}\right) u\right]=a_{12}(x) v-a_{11}(x) u, & x \in \Omega, \\ -\Delta\left[\left(d_{2}+\frac{\beta_{2}}{\gamma_{2}+u}+\alpha_{2} v\right) v\right]=g(u)-a_{22}(x) v, & x \in \Omega, \\ u(x)=v(x)=0, & x \in \partial \Omega .\end{cases}
$$

Mathematically, the solution $(u, v)$ to problem (1.4) is called a coexistence if $u>0$ and $v>0$ for every $x \in \Omega$.

The rest of this paper is organized as follows. Section 2 is devoted to introducing the basic reproduction number of epidemic model (1.3) by using the next infection operator and corresponding eigenvalue problem. The sufficient condition for the existence and nonexistence of the coexistence solution are presented in Section 2. Finally, some numerical simulations which confirm our analytical findings, as well as a brief discussion, are present in Section 4.

\section{The basic reproduction numbers}

In this section, we will introduce the basic reproduction number and analyze its properties for the corresponding elliptic system (1.4) in $\Omega$. We linearize the first two equations around disease-free equilibrium $(0,0)$ to obtain the following linear cooperative system:

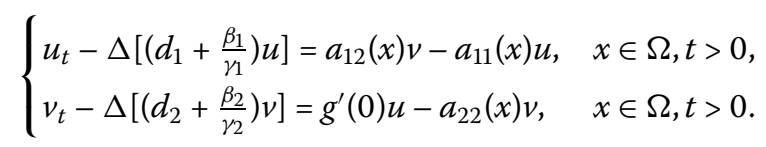

The basic reproduction number $R_{0}$ is defined as the expected number of secondary cases produced, in a completely susceptible population, by a typical infected individual during its entire period of infectiousness [21]. In order to introduce the basic reproduction number for the diffusive epidemic model (2.1), as a first step, we need to define the next infection operator for problem (2.1). Similarly as in [22], we set $X_{1}:=\left(C\left(\bar{\Omega}, R^{2}\right)\right)^{2}$ and $X_{1}^{+}:=\left(C\left(\bar{\Omega}, R_{+}^{2}\right)\right)^{2}$. Let $T(t)$ be the solution semigroup on $X_{1}$ associated with the linear system

$$
\begin{cases}u_{t}-\Delta\left[\left(d_{1}+\frac{\beta_{1}}{\gamma_{1}}\right) u\right]=-a_{11}(x) u, & x \in \Omega, t>0, \\ v_{t}-\Delta\left[\left(d_{2}+\frac{\beta_{2}}{\gamma_{2}}\right) v\right]=-a_{22}(x) v, & x \in \Omega, t>0 .\end{cases}
$$

Define

$$
L(\phi)(x):=\int_{0}^{\infty} F(x)[T(t) \phi](x) d t=F(x) \int_{0}^{\infty}[T(t) \phi](x) d t,
$$


where

$$
F(x):=\left(\begin{array}{cc}
0 & a_{12}(x) \\
g^{\prime}(0) & 0
\end{array}\right)
$$

and $F(x)[T(t) \phi](x)$ accounts for the distribution of new infective members at time $t$. The continuous and positive operator $L$, called the next generation operator, represents the density distribution of the accumulative new infections at location $x$. As stated in [22], we define the spectral radius of $L$ as the basic reproduction number

$$
R_{0}^{D}:=\rho(L)
$$

for the problem (2.1).

As discussed in [23], we can proved that $R_{0}^{D}$ is the principal eigenvalue of the associated linearized eigenvalue problem

$$
\begin{cases}-\left(d_{1}+\frac{\beta_{1}}{\gamma_{1}}\right) \Delta \phi=\frac{a_{12}(x)}{R_{D}^{D}} \psi-a_{11}(x) \phi, & x \in \Omega, \\ -\left(d_{2}+\frac{\beta_{2}}{\gamma_{2}}\right) \Delta \psi=\frac{g^{\prime}(0)}{R_{0}^{D}} \phi-a_{22}(x) \psi, & x \in \Omega, \\ \phi(x)=\psi(x)=0, & x \in \partial \Omega .\end{cases}
$$

The eigenfunction pair $(\phi, \psi)$ with $\phi>0$ and $\psi>0$ in $\Omega$ is unique (subject to constant multiples). Moreover, $R_{0}^{D}$ is algebraically simple and dominant, and it possesses the following analytical properties.

Theorem 2.1 $\operatorname{sign}\left(1-R_{0}^{D}\right)=\operatorname{sign}\left(\lambda_{0}\right)$, where $\lambda_{0}$ is the principal eigenvalue of the eigenvalue problem

$$
\begin{cases}-\left(d_{1}+\frac{\beta_{1}}{\gamma_{1}}\right) \Delta \phi=a_{12}(x) \psi-a_{11}(x) \phi+\lambda_{0} \phi, & x \in \Omega, \\ -\left(d_{2}+\frac{\beta_{2}}{\gamma_{2}}\right) \Delta \psi=g^{\prime}(0) \phi-a_{22}(x) \psi+\lambda_{0} \psi, & x \in \Omega, \\ \phi(x)=\psi(x)=0, & x \in \partial \Omega .\end{cases}
$$

Proof For convenience, let

$$
B:=\left(\begin{array}{cc}
\Delta\left(d_{1}+\frac{\beta_{1}}{\gamma_{1}}\right) & 0 \\
0 & \Delta\left(d_{2}+\frac{\beta_{2}}{\gamma_{2}}\right)
\end{array}\right)-\left(\begin{array}{cc}
a_{11}(x) & 0 \\
0 & a_{22}(x)
\end{array}\right) .
$$

One can easily prove that $B$ is the generator of the semigroup $T(t)$ on $X_{1}$. Due to $T(t)$ is a positive semigroup, that is, $T(t) X_{1}^{+} \subseteq X_{1}^{+}$for all $t \geq 0$, it follows from [24] that the operator is resolvent-positive, and

$$
(\lambda I-B)^{-1} \phi=\int_{0}^{\infty} e^{-\lambda t} T(t) \phi d t, \quad \forall \lambda>\lambda^{*}, \phi \in X_{1},
$$

where $\lambda^{*}$ is the principal eigenvalue of problem (2.2). Letting $\lambda=0$, we obtain

$$
-B^{-1} \phi=\int_{0}^{\infty} T(t) \phi d t, \quad \forall \phi \in X_{1},
$$


then we derive that $L=-F B^{-1}$. Denote the linear operator $A:=B+F$, it is easy to deduce that $\lambda_{0}$ is the principal eigenvalue of the operator $A$. Thus, it follows from [24] that $\lambda_{0}$ has the same sign as $1-\rho\left(-F B^{-1}\right)=1-R_{0}^{D}$.

Remark 2.1 It is well known that $\lambda_{0}$ is monotonically decreasing with respect to $a_{12}(x)$, and we deduce from Theorem 2.1 that $R_{0}^{D}$ is monotonically increasing with respect to $a_{12}(x)$, thus $R_{0}^{D}>1$ if $a_{12}(x)$ is sufficiently large.

When all coefficients in problem (1.3) are constants, we shall present an explicit formula for $R_{0}^{D}$, which is in line with the basic reproduction number for the corresponding fecallyorally epidemic model with homogeneous boundary condition in a fixed region [22, 25].

Theorem 2.2 If $a_{11}(x)=a_{11}^{*}, a_{12}(x)=a_{12}^{*}$ and $a_{22}(x)=a_{22}^{*}$, then the principal eigenvalue $R_{0}^{D}$ for (2.4), or the basic reproduction number for model (1.3), is represented by

$$
R_{0}^{D}(\Omega)=\sqrt{\frac{a_{12}^{*} g^{\prime}(0)}{\left[D_{1}^{*} \lambda^{*}+a_{11}^{*}\right]\left[D_{2}^{*} \lambda^{*}+a_{22}^{*}\right]}},
$$

where $\left(d_{1}+\frac{\beta_{1}}{\gamma_{1}}\right)=D_{1}^{*},\left(d_{2}+\frac{\beta_{2}}{\gamma_{2}}\right)=D_{2}^{*}$, and $\lambda^{*}$ is the principal eigenvalue of $-\Delta$ in $\Omega$ with homogeneous Dirichlet boundary condition.

Proof Let $\psi^{*}$ be the eigenfunction to corresponding the principal eigenvalue $\left(\lambda^{*}\right)$ of $-\Delta$ in $\Omega$ with homogeneous Dirichlet boundary condition, that is, there exists a function $\psi^{*}(x)>$ 0 satisfying

$$
\begin{cases}-\Delta \psi^{*}=\lambda^{*} \psi^{*}, & x \in \Omega, \\ \psi^{*}=0, & x \in \partial \Omega\end{cases}
$$

and take

$$
\begin{aligned}
A^{*} & =\frac{a_{12}^{*} g^{\prime}(0)}{\left[D_{1}^{*} \lambda^{*}+a_{11}^{*}\right]\left[D_{2}^{*} \lambda^{*}+a_{22}^{*}\right]}, \\
\phi^{*} & =\frac{a_{12}^{*}}{\sqrt{R^{*}}\left[D_{1}^{*} \lambda^{*}+a_{11}^{*}\right]} \psi^{*} .
\end{aligned}
$$

Straightforward computations show that the eigenfunction pair $\left(\phi^{*}, \psi^{*}\right)$ is a positive solution to problem (2.4) when $R_{0}^{D}=\sqrt{A^{*}}$. Due to the uniqueness of the principal eigenvalue of (2.4), one can obtain (2.7) immediately.

\section{Coexistence}

In this section, inspired by $[14,16,26]$, we first study the existence of a coexistence solution to problem (1.3) by constructing upper and lower solutions. For the convenience, we let

$$
\begin{aligned}
& f_{1}(x, u, v)=a_{12}(x) v-a_{11}(x) u, \\
& f_{2}(x, u, v)=g(u)-a_{22}(x) v, \\
& S=\left\{(u, v) \in(\mathcal{C}(\bar{\Omega}))^{2} ;(\hat{u}, \hat{v}) \leq(u, v) \leq(\tilde{u}, \tilde{v}), x \in \bar{\Omega}\right\},
\end{aligned}
$$

where $(\hat{u}, \hat{v})$ and $(\tilde{u}, \tilde{v})$ are given in the following definition. 
As stated in [16], we are now in a position to present a sufficient condition for which system (1.3) possesses a positive solution by constructing suitable upper and lower solutions. For the sake of simplicity, we first give an equivalent form of problem (1.3):

$$
\begin{cases}-\Delta\left[H_{1}(u, v)\right]=f_{1}(x, u, v), & x \in \Omega, \\ -\Delta\left[H_{2}(u, v)\right]=f_{2}(x, u, v), & x \in \Omega, \\ u(x)=v(x)=0, & x \in \partial \Omega,\end{cases}
$$

where $H_{1}(u, v)=\left(d_{1}+\alpha_{1} u+\frac{\beta_{1}}{\gamma_{1}+v}\right) u, H_{2}(u, v)=\left(d_{2}+\alpha_{2} v+\frac{\beta_{2}}{\gamma_{2}+u}\right) v$.

Taking $w=H_{1}(u, v)$ and $z=H_{2}(u, v)$, then the Jacobian determinant of the transformation $(u, v) \rightarrow(w, z)$ is given by

$$
\begin{aligned}
J= & \frac{\partial(w, z)}{\partial(u, v)} \\
= & \left(d_{1}+2 \alpha_{1} u+\frac{\beta_{1}}{\gamma_{1}+v}\right)\left(d_{2}+\frac{\beta_{2}}{\gamma_{2}+u}+2 \alpha_{2} v\right)-\frac{\beta_{1} u}{\left(\gamma_{1}+v\right)^{2}} \frac{\beta_{2} v}{\left(\gamma_{2}+u\right)^{2}} \\
= & d_{1} d_{2}+2\left(\alpha_{2} d_{1} v+\alpha_{1} d_{2} u\right)+4 \alpha_{1} \alpha_{2} u v+2\left(\frac{\alpha_{1} \beta_{2} u}{\gamma_{2}+u}+\frac{\alpha_{2} \beta_{1} v}{\gamma_{1}+v}\right) \\
& +\left(\frac{d_{1} \beta_{2}}{\gamma_{2}+u}+\frac{d_{2} \beta_{1}}{\gamma_{1}+v}\right)+\frac{\beta_{1} \beta_{2}}{\left(\gamma_{1}+v\right)\left(\gamma_{2}+u\right)}-\frac{\beta_{1} u}{\left(\gamma_{1}+v\right)^{2}} \frac{\beta_{2} v}{\left(\gamma_{2}+u\right)^{2}} \\
> & d_{1} d_{2}>0 \quad \text { for }(u, v) \geq(0,0) .
\end{aligned}
$$

Therefore, the inverse functions $u=g_{1}(w, z), v=g_{2}(w, z)$ exist as long as $(u, v) \geq(0,0)$. Hence, problem (1.4) reduces to the following equivalent form:

$$
\begin{cases}-\Delta w+k_{1} w=F_{1}(x, u, v), & x \in \Omega, \\ -\Delta z+k_{2} z=F_{2}(x, u, v), & x \in \Omega, \\ u=g_{1}(w, z), \quad v=g_{2}(w, z), & x \in \Omega, \\ w(x)=z(x)=0, & x \in \partial \Omega,\end{cases}
$$

where $F_{i}(x, u, v)=k_{i} H_{i}(u, v)+f_{i}(x, u, v), i=1,2$, and the constants $k_{1}, k_{2}$ will be chosen later to make sure that $F_{1}$ and $F_{2}$ are increasing with respect to $u$ and $v$, respectively.

On the other hand, an elementary computation yields

$$
\frac{\partial u}{\partial w} \geq 0, \quad \frac{\partial u}{\partial z} \geq 0, \quad \frac{\partial v}{\partial w} \geq 0, \quad \frac{\partial v}{\partial z} \geq 0,
$$

which implies that $u=g_{1}(w, z)$ is nondecreasing in both $w$ and $z$, and $v=g_{2}(w, z)$ is also nondecreasing in both $u$ and $v$ for all $(u, v) \geq(0,0)$. Since problem (3.2) is quasi-monotone nondecreasing, then we can define the ordered upper and lower solutions to problem (3.2) as follows.

Definition 3.1 Assume that $F_{1}$ and $F_{2}$ are increasing with respect to $u$ and $v$. A pair of 4nonnegative functions $(\tilde{u}, \tilde{v}, \tilde{w}, \tilde{z}),(\hat{u}, \hat{v}, \hat{w}, \hat{z})$ in $\mathcal{C}^{2}(\Omega) \cap \mathcal{C}(\bar{\Omega})$ are called ordered upper and 
lower solutions of (3.2), if

$$
(0,0) \leq(\hat{u}, \hat{v}) \leq(\tilde{u}, \tilde{v}), \quad(0,0) \leq(\hat{w}, \hat{z}) \leq(\tilde{w}, \tilde{z})
$$

and

$$
\begin{cases}-\Delta \tilde{w}+k_{1} \tilde{w} \geq F_{1}(x, \tilde{u}, \tilde{v}), & x \in \Omega, \\ -\Delta \tilde{z}+k_{2} \tilde{z} \geq F_{2}(x, \tilde{u}, \tilde{v}), & x \in \Omega, \\ -\Delta \hat{w}+k_{1} \hat{w} \leq F_{1}(x, \hat{u}, \hat{v}), & x \in \Omega, \\ -\Delta \hat{z}+k_{1} \hat{z} \leq F_{2}(x, \hat{u}, \hat{v}), & x \in \Omega, \\ \tilde{u} \geq g_{1}(\tilde{w}, \tilde{z}), \quad \hat{u} \leq g_{1}(\hat{w}, \hat{z}), & x \in \Omega, \\ \tilde{v} \geq g_{2}(\tilde{w}, \tilde{z}), \quad \hat{v} \leq g_{2}(\hat{w}, \hat{z}), & x \in \Omega, \\ \tilde{w}(x) \geq 0 \geq \hat{w}(x), \quad \tilde{z}(x) \geq 0 \geq \hat{z}(x), & x \in \partial \Omega .\end{cases}
$$

For consistency, we denote

$$
\tilde{u}=g_{1}(\tilde{w}, \tilde{z}), \quad \tilde{v}=g_{2}(\tilde{w}, \tilde{z}), \quad \hat{u}=g_{1}(\hat{w}, \hat{z}), \quad \hat{v}=g_{2}(\hat{w}, \hat{z}),
$$

which means that

$$
\tilde{w}=H_{1}(\tilde{u}, \tilde{v}), \quad \tilde{z}=H_{2}(\tilde{u}, \tilde{v}), \quad \hat{w}=H_{1}(\hat{u}, \hat{v}), \quad \hat{v}=H_{2}(\hat{u}, \hat{v}) .
$$

Then (3.3) is rewritten as follows:

$$
\begin{cases}-\Delta\left[\left(d_{1}+\alpha_{1} \tilde{u}+\frac{\beta_{1}}{\gamma_{1}+\tilde{v}}\right) \tilde{u}\right] \geq a_{12}(x) \tilde{v}-a_{11}(x) \tilde{u}, & x \in \Omega, \\ -\Delta\left[\left(d_{2}+\alpha_{2} \tilde{v}+\frac{\beta_{2}}{\gamma_{2}+\tilde{u}}\right) \tilde{v}\right] \geq g(\tilde{u})-a_{22}(x) \tilde{v}, & x \in \Omega, \\ -\Delta\left[\left(d_{1}+\alpha_{1} \hat{u}+\frac{\beta_{1}}{\gamma_{1}+\hat{v}}\right) \hat{u}\right] \leq a_{12}(x) \hat{v}-a_{11}(x) \hat{u}, & x \in \Omega, \\ -\Delta\left[\left(d_{2}+\alpha_{2} \hat{v}+\frac{\beta_{2}}{\gamma_{2}+\hat{u}}\right) \hat{v}\right] \leq g(\hat{u})-a_{22}(x) \hat{v}, & x \in \Omega, \\ \tilde{u}(x) \geq 0 \geq \hat{u}(x), \quad \tilde{v}(x) \geq 0 \geq \hat{v}(x), & x \in \partial \Omega .\end{cases}
$$

The pairs $(\tilde{u}, \tilde{v})$ and $(\hat{u}, \hat{v})$, which satisfy $(3.4)$ and $(\hat{u}, \hat{v}) \leq(\tilde{u}, \tilde{v})$, are also called ordered upper and lower solutions of (1.4), respectively.

Theorem 3.1 Assume that $R_{0}^{D}>1$, and $\frac{\beta_{1}}{\gamma_{1}^{2}}, \frac{\beta_{2}}{\gamma_{2}^{2}}$ are sufficiently small, then problem (1.3) admits at least one coexistence solution $(u(x), v(x))$.

Proof To verify the existence of a positive solution to problem (1.4), it suffices to find a pair of upper and lower solutions to problem (1.4). We seek such as in the form $(\tilde{u}, \tilde{v})=\left(M_{1}, M_{2}\right),(\hat{u}, \hat{v})=\left(g_{1}\left(\delta\left(d_{1}+\frac{\beta_{1}}{\gamma_{1}}\right) \phi, \delta\left(d_{2}+\frac{\beta_{2}}{\gamma_{2}}\right) \psi\right), g_{2}\left(\delta\left(d_{1}+\frac{\beta_{1}}{\gamma_{1}}\right) \phi, \delta\left(d_{2}+\frac{\beta_{2}}{\gamma_{2}}\right) \psi\right)\right)$, where $M_{i}(i=1,2)$ and $\delta$ are some positive constants with $\delta$ small enough, $(\phi, \psi) \equiv(\phi(x), \psi(x))$ is (normalized) positive eigenfunction corresponding to $\lambda_{0}$, and $\lambda_{0}$ is the principal eigenvalue of the linear eigenvalue problem (2.5). 
In fact, $\left(M_{1}, M_{2}\right)$ and $\left(g_{1}\left(\delta\left(d_{1}+\frac{\beta_{1}}{\gamma_{1}}\right) \phi, \delta\left(d_{2}+\frac{\beta_{2}}{\gamma_{2}}\right) \psi\right), g_{2}\left(\delta\left(d_{1}+\frac{\beta_{1}}{\gamma_{1}}\right) \phi, \delta\left(d_{2}+\frac{\beta_{2}}{\gamma_{2}}\right) \psi\right)\right)$ satisfy the inequalities in (3.4) if the following inequalities:

$$
\left\{\begin{array}{l}
-\Delta\left[\left(d_{1}+\alpha_{1} M_{1}+\frac{\beta_{1}}{\gamma_{1}+M_{2}}\right) M_{1}\right] \geq a_{12}(x) M_{2}-a_{11}(x) M_{1}, \\
-\Delta\left[\left(d_{2}+\frac{\beta_{2}}{\gamma_{2}+M_{1}}+\alpha_{2} M_{2}\right) M_{2}\right] \geq g\left(M_{1}\right)-a_{22}(x) M_{2}, \\
-\Delta\left[\left(d_{1}+\frac{\beta_{1}}{\gamma_{1}}\right) \phi\right] \\
\quad \leq a_{12}(x)\left(d_{2}+\frac{\beta_{2}}{\gamma_{2}}\right) \psi /\left(d_{2}+\alpha_{2} \hat{v}+\frac{\beta_{2}}{\gamma_{2}+\hat{u}}\right)-a_{11}(x)\left(d_{1}+\frac{\beta_{1}}{\gamma_{1}}\right) \phi /\left(d_{1}+\alpha_{1} \hat{u}+\frac{\beta_{1}}{\gamma_{1}+\hat{v}}\right), \\
-\Delta\left[\left(d_{2}+\frac{\beta_{2}}{\gamma_{2}}\right) \psi\right] \\
\quad \leq g^{\prime}(\xi)\left(d_{1}+\frac{\beta_{1}}{\gamma_{1}}\right) \phi /\left(d_{1}+\alpha_{1} \hat{u}+\frac{\beta_{1}}{\gamma_{1}+\hat{v}}\right)-a_{22}(x)\left(d_{2}+\frac{\beta_{2}}{\gamma_{2}}\right) \psi /\left(d_{2}+\alpha_{2} \hat{v}+\frac{\beta_{2}}{\gamma_{2}+\hat{u}}\right),
\end{array}\right.
$$

hold, where $\xi \in(0, \hat{u})$.

On one hand, due to $\limsup _{z \rightarrow \infty} \frac{g(z)}{z}<\min _{\bar{\Omega}}\left\{\frac{a_{11}(x)}{a_{12}(x)}\right\} \cdot \min _{\bar{\Omega}}\left\{a_{22}(x)\right\}$, there exists constant $M_{0}$ such that $\frac{g(z)}{z}<\min _{\bar{\Omega}}\left\{\frac{a_{11}(x)}{a_{12}(x)}\right\} \cdot \min _{\bar{\Omega}}\left\{a_{22}(x)\right\}$ for $z \geq M_{0}$. As a result, the first two inequalities in (3.5) will hold if we set $(\tilde{u}, \tilde{v})=\left(M_{1}, M_{2}\right)$, where $M_{1}=\max \left\{M_{0}, \max _{\bar{\Omega}} u(x, 0)\right.$, $\left.\max _{\bar{\Omega}} v(x, 0)\right\}, M_{2}=M_{1} \cdot \max _{\bar{\Omega}}\left\{\frac{a_{12}(x)}{a_{11}(x)}\right\}$.

On the other hand, we can derive the relations

$$
\delta\left(d_{1}+\frac{\beta_{1}}{\gamma_{1}}\right) \phi=\left(d_{1}+\alpha_{1} \hat{u}+\frac{\beta_{1}}{\gamma_{1}+\hat{v}}\right) \hat{u}, \delta\left(d_{2}+\frac{\beta_{2}}{\gamma_{2}}\right) \psi=\left(d_{2}+\alpha_{2} \hat{v}+\frac{\beta_{2}}{\gamma_{2}+\hat{u}}\right) \hat{v}
$$

which imply that $0<\hat{u} \leq \delta\left(1+\beta_{1} /\left(d_{1} \gamma_{1}\right)\right) \phi$ and $0<\hat{v} \leq \delta\left(1+\beta_{2} /\left(d_{2} \gamma_{2}\right)\right) \psi$.

In view of (2.5), the last two inequalities in (3.5) can be simplified as follows:

$$
\left\{\begin{array}{l}
a_{12}(x) \psi-a_{11}(x) \phi+\lambda_{0} \phi \\
\quad \leq a_{12}(x)\left(d_{2}+\frac{\beta_{2}}{\gamma_{2}}\right) \psi /\left(d_{2}+\alpha_{2} \hat{v}+\frac{\beta_{2}}{\gamma_{2}+\hat{u}}\right)-a_{11}(x)\left(d_{1}+\frac{\beta_{1}}{\gamma_{1}}\right) \phi /\left(d_{1}+\alpha_{1} \hat{u}+\frac{\beta_{1}}{\gamma_{1}+\hat{v}}\right) \\
g^{\prime}(0) \phi-a_{22}(x) \psi+\lambda_{0} \psi \\
\quad \leq g^{\prime}(\xi)\left(d_{1}+\frac{\beta_{1}}{\gamma_{1}}\right) \phi /\left(d_{1}+\alpha_{1} \hat{u}+\frac{\beta_{1}}{\gamma_{1}+\hat{v}}\right)-a_{22}(x)\left(d_{2}+\frac{\beta_{2}}{\gamma_{2}}\right) \psi /\left(d_{2}+\alpha_{2} \hat{v}+\frac{\beta_{2}}{\gamma_{2}+\hat{u}}\right) .
\end{array}\right.
$$

Since $R_{0}^{D}>1$, we can deduce that the principal eigenvalue $\lambda_{0}$ of problem (2.5) satisfies $\lambda_{0}<0$ by applying Theorem 2.1. Therefore we can choose $\delta$ sufficiently small such that (3.6) hold.

Due to

$$
\begin{aligned}
& \frac{\partial F_{1}}{\partial u}=k_{1}\left(d_{1}+2 \alpha_{1} u+\frac{\beta_{1}}{\gamma_{1}+v}\right)-a_{11}(x), \\
& \frac{\partial F_{1}}{\partial v}=\frac{-k_{1} u \beta_{1}}{\left(\gamma_{1}+v\right)^{2}}+a_{12}(x),
\end{aligned}
$$

we choose $k_{1}=\frac{a_{11}^{M}}{d_{1}}$, if $\frac{\beta_{1}}{\gamma_{1}^{2}} \leq \frac{d_{1} a_{12}^{m}}{a_{11}^{M}}$, then $F_{1}$ is increasing with respect to $u$ and $v$, respectively, where $a_{11}^{M}=\max _{x \in \bar{\Omega}} a_{11}(x)$ and $a_{12}^{m}=\min _{x \in \bar{\Omega}} a_{12}(x)$. Analogously, we also choose $k_{2}=\frac{a_{22}^{M}}{d_{2}}$, if $\frac{\beta_{2}}{\gamma_{2}^{2}} \leq \frac{d_{2} g^{\prime}(0)}{a_{22}^{M}}$, then $F_{2}$ is increasing with respect to $u$ and $v$, respectively, where $a_{22}^{M}=\max _{x \in \bar{\Omega}} a_{22}(x)$.

Consequently, the pair $(\tilde{u}, \tilde{v})=\left(M_{1}, M_{2}\right),(\hat{u}, \hat{v})=\left(g_{1}\left(\delta\left(d_{1}+\frac{\beta_{1}}{\gamma_{1}}\right) \phi, \delta\left(d_{2}+\frac{\beta_{2}}{\gamma_{2}}\right) \psi\right), g_{2}\left(\delta\left(d_{1}+\right.\right.\right.$ $\left.\left.\left.\frac{\beta_{1}}{\gamma_{1}}\right) \phi, \delta\left(d_{2}+\frac{\beta_{2}}{\gamma_{2}}\right) \psi\right)\right)$ are ordered upper and lower solutions to problem (1.4), respectively. 
Applying Theorem 2.1 of [16] leads to the existence of the coexistence solution to problem (1.4).

In what follows, we will present the non-existence result of any coexistence steady state.

Theorem 3.2 If $R_{0}^{D}<1$, problem (1.3) has no positive steady-state solution.

Proof Suppose $\left(u^{*}(x), v^{*}(x)\right)$ is a coexistence solution to problem (1.3) by contradiction. It follows from the strong maximum principle that $\frac{\partial u^{*}}{\partial \eta}(x)<0$ and $\frac{\partial \nu^{*}}{\partial \eta}(x)<0$ for $x \in \partial \Omega$, therefore, there exist $M>0$ and $m>0$ such that

$$
m v^{*}(x) \leq u^{*}(x) \leq M v^{*}(x) \quad \text { for } x \in \bar{\Omega}
$$

We now consider the following linear eigenvalue problem:

$$
\begin{cases}-\left(d_{1}+\frac{\beta_{1}}{\gamma_{1}}\right) \Delta \phi=a_{12}(x) \psi-a_{11}(x) \phi+\mu \phi, & x \in \Omega, \\ -\left(d_{2}+\frac{\beta_{2}}{\gamma_{2}}\right) \Delta \psi=g^{\prime}(0) \phi-a_{22}(x) \psi+\mu \psi, & x \in \Omega, \\ \phi(x)=\psi(x)=0, & x \in \partial \Omega .\end{cases}
$$

Next, we shall claim that the principal eigenvalue $\mu \leq 0$, which results in $R_{0}^{D} \geq 1$. This leads to a contradiction.

To prove $\mu \leq 0$, we set

$$
\begin{aligned}
& \phi^{*}=\frac{1}{\varepsilon}\left(d_{1}+\alpha_{1} u^{*}+\frac{\beta_{1}}{\gamma_{1}+v^{*}}\right) u^{*} /\left(d_{1}+\frac{\beta_{1}}{\gamma_{1}}\right), \\
& \psi^{*}=\frac{1}{\varepsilon}\left(d_{2}+\alpha_{2} v^{*}+\frac{\beta_{2}}{\gamma_{2}+u^{*}}\right) v^{*} /\left(d_{2}+\frac{\beta_{2}}{\gamma_{2}}\right),
\end{aligned}
$$

then (1.3) is equivalent to the following problem:

$$
\begin{cases}-\left(d_{1}+\frac{\beta_{1}}{\gamma_{1}}\right) \Delta \phi^{*}=a_{12}(x) \psi^{*}-a_{11}(x) \phi^{*}+F \phi^{*}, & x \in \Omega, \\ -\left(d_{2}+\frac{\beta_{2}}{\gamma_{2}}\right) \Delta \psi^{*}=g^{\prime}(0) \phi^{*}-a_{22}(x) \psi^{*}+G \psi^{*}, & x \in \Omega, \\ \phi^{*}(x)=\psi^{*}(x)=0, & x \in \partial \Omega,\end{cases}
$$

where

$$
\begin{aligned}
& F=a_{12}(x)\left(H_{2}-1\right) \frac{\psi^{*}}{\phi^{*}}-a_{11}(x)\left(H_{1}-1\right)-a_{12}(x) H_{1} H_{2} \varepsilon \psi^{*}, \\
& G=g^{\prime}(0)\left(H_{1}-1\right) \frac{\phi^{*}}{\psi^{*}}-a_{22}(x)\left(H_{2}-1\right)-g^{\prime}(0) H_{1} H_{2} \varepsilon \phi^{*}, \\
& H_{1}(x)=\frac{d_{1}+\beta_{1} / \gamma_{1}}{d_{1}+\alpha_{1} u^{*}+\beta_{1} /\left(\gamma_{1}+v^{*}\right)}, \quad H_{2}(x)=\frac{d_{2}+\beta_{2} / \gamma_{2}}{d_{2}+\alpha_{2} v^{*}+\beta_{2} /\left(\gamma_{2}+u^{*}\right)} .
\end{aligned}
$$

From the above, we can obtain

$$
u^{*} \leq \varepsilon \phi^{*}\left[1+\beta_{1} /\left(\gamma_{1} d_{1}\right)\right], \quad v^{*} \leq \varepsilon \psi^{*}\left[1+\beta_{2} /\left(\gamma_{2} d_{2}\right)\right], \quad m \leq \frac{u^{*}}{v^{*}} \leq M
$$


therefore there exists a positive constant $M^{*}$ such that $|F| \leq M^{*} \varepsilon$ and $|G| \leq M^{*} \varepsilon$. Then (3.8) can be rewritten as

$$
\begin{cases}-\left(d_{1}+\frac{\beta_{1}}{\gamma_{1}}\right) \Delta \phi^{*}=a_{12}(x)(x) \psi^{*}-a_{11}(x) \phi^{*}+\left(-M^{*} \varepsilon+F\right) \phi^{*}+M^{*} \varepsilon \phi^{*}, & x \in \Omega, \\ -\left(d_{2}+\frac{\beta_{2}}{\gamma_{2}}\right) \Delta \psi^{*}=g^{\prime}(0) \phi^{*}-a_{22}(x) \psi^{*}+\left(-M^{*} \varepsilon+G\right) \psi^{*}+M^{*} \varepsilon \psi^{*}, & x \in \Omega, \\ \phi^{*}(x)=\psi^{*}(x)=0, & x \in \partial \Omega .\end{cases}
$$

Comparing (3.7) and (3.9), together with the monotonicity of $\mu$ with respect to the coefficients in (3.7), we can easily derive $\mu \leq \varepsilon M^{*}$, which implies that $\mu \leq 0$, since $\varepsilon$ is arbitrarily small. This completes the proof.

Remark 3.1 Assume that all coefficients of (1.3) are spatially independent. $R_{0}^{D}$ is represented by (2.7). If the cross-diffusion coefficients, i.e., $\frac{\beta_{1}}{\gamma_{1}}$ or $\frac{\beta_{2}}{\gamma_{2}}$ is large enough, then no coexistence solution to problem (1.3) exists.

In what follows, we employ the monotone iterative schemes to construct the true solutions of (1.4). It follows from $R_{0}^{D}>1$ that $\left(M_{1}, M_{2}\right)$ and $\left(g_{1}\left(\delta\left(d_{1}+\frac{\beta_{1}}{\gamma_{1}}\right) \phi, \delta\left(d_{2}+\frac{\beta_{2}}{\gamma_{2}}\right) \psi\right), g_{2}\left(\delta\left(d_{1}+\right.\right.\right.$ $\left.\left.\left.\frac{\beta_{1}}{\gamma_{1}}\right) \phi, \delta\left(d_{2}+\frac{\beta_{2}}{\gamma_{2}}\right) \psi\right)\right)$ are ordered upper and lower solution to problem (1.3), respectively. Selecting $\left(\bar{u}^{(0)}, \bar{v}^{(0)}\right)=\left(M_{1}, M_{2}\right)$ and $\left(\underline{u}^{(0)}, \underline{v}^{(0)}\right)=\left(g_{1}\left(\delta\left(d_{1}+\frac{\beta_{1}}{\gamma_{1}}\right) \phi, \delta\left(d_{2}+\frac{\beta_{2}}{\gamma_{2}}\right) \psi\right), g_{2}\left(\delta\left(d_{1}+\right.\right.\right.$ $\left.\left.\left.\frac{\beta_{1}}{\gamma_{1}}\right) \phi, \delta\left(d_{2}+\frac{\beta_{2}}{\gamma_{2}}\right) \psi\right)\right)$ as an initial iteration, we can construct a sequence $\left\{\left(u^{(n)}, v^{(n)}\right)\right\}$ from the iteration process

$$
\begin{cases}-\Delta \bar{w}^{(n)}+k_{1} \bar{w}^{(n)}=F_{1}\left(x, \bar{u}^{(n-1)}, \bar{v}^{(n-1)}\right), & x \in \Omega, \\ -\Delta \bar{z}^{(n)}+k_{2} \bar{z}^{(n)}=F_{2}\left(x, \bar{u}^{(n-1)}, \bar{v}^{(n-1)}\right), & x \in \Omega, \\ -\Delta \underline{w}^{(n)}+k_{1} \underline{w}^{(n)}=F_{1}\left(x, \underline{u}^{(n-1)}, \underline{v}^{(n-1)}\right), & x \in \Omega, \\ -\Delta \underline{z}^{(n)}+k_{2} \underline{z}^{(n)}=F_{2}\left(x, \underline{u}^{(n-1)}, \underline{v}^{(n-1)}\right), & x \in \Omega, \\ \bar{u}^{(n)}=g_{1}\left(\bar{w}^{(n)}, \bar{z}^{(n)}\right), \quad \underline{u}^{(n)}=g_{1}\left(\underline{w}^{(n)}, \underline{z}^{(n)}\right), & x \in \Omega, \\ \bar{v}^{(n)}=g_{2}\left(\bar{w}^{(n)}, \bar{z}^{(n)}\right), \quad \underline{v}^{(n)}=g_{2}\left(\underline{w}^{(n)}, \underline{z}^{(n)}\right), & x \in \Omega, \\ \bar{w}^{(n)}(x)=\underline{w}^{(n)}(x)=0, \quad \bar{z}^{(n)}(x)=\underline{z}^{(n)}(x)=0, & x \in \partial \Omega,\end{cases}
$$

where $n=1,2, \ldots$.

As in Lemma 3.1 in [16], the sequences $\left\{\left(\bar{w}^{(n)}, \bar{z}^{(n)}\right)\right\}$ and $\left\{\left(\underline{w}^{(n)}, \underline{z}^{(n)}\right)\right\}$ governed by (3.10) are well-posed and possess the monotone property,

$$
\begin{aligned}
(\hat{w}, \hat{z}) & \leq\left(\underline{w}^{(n-1)}, \underline{z}^{(n-1)}\right) \leq\left(\underline{w}^{(n)}, \underline{z}^{(n)}\right) \leq\left(\bar{w}^{(n)}, \bar{z}^{(n)}\right) \\
& \leq\left(\bar{w}^{(n-1)}, \bar{z}^{(n-1)}\right) \leq(\tilde{w}, \tilde{z}) \quad \text { for } n=1,2, \ldots
\end{aligned}
$$

Consequently, the pointwise limits

$$
\lim _{n \rightarrow \infty}\left(\bar{w}^{(n)}, \bar{z}^{(n)}\right)=(\bar{w}, \bar{z}), \quad \lim _{n \rightarrow \infty}\left(\underline{w}^{(n)}, \underline{z}^{(n)}\right)=(\underline{w}, \underline{z})
$$

exist and their limits obey the relations

$$
(\hat{w}, \hat{z}) \leq\left(\underline{w}^{(n)}, \underline{z}^{(n)}\right) \leq(\underline{w}, \underline{z}) \leq(\bar{w}, \bar{z}) \leq\left(\bar{w}^{(n)}, \bar{z}^{(n)}\right) \leq(\tilde{w}, \tilde{z})
$$

for every $n=1,2, \ldots$. 
The last three equations in (3.10) imply

$$
\begin{array}{ll}
\bar{u}^{(n)}=g_{1}\left(\bar{w}^{(n)}, \bar{z}^{(n)}\right), & \underline{u}^{(n)}=g_{1}\left(\underline{w}^{(n)}, \underline{z}^{(n)}\right), \\
\bar{v}^{(n)}=g_{2}\left(\bar{w}^{(n)}, \bar{z}^{(n)}\right), & \underline{v}^{(n)}=g_{2}\left(\underline{w}^{(n)}, \underline{z}^{(n)}\right),
\end{array}
$$

which is equivalent to

$$
\begin{array}{ll}
\bar{w}^{(n)}=H_{1}\left(\bar{u}^{(n)}, \bar{v}^{(n)}\right), & \underline{w}^{(n)}=H_{1}\left(\underline{u}^{(n)}, \underline{v}^{(n)}\right), \\
\bar{z}^{(n)}=H_{2}\left(\bar{u}^{(n)}, \bar{v}^{(n)}\right), & \underline{z}^{(n)}=H_{2}\left(\underline{u}^{(n)}, \underline{v}^{(n)}\right) .
\end{array}
$$

Letting $n \rightarrow \infty$ in (3.10) and applying the standard regularity theory and compact argument for elliptic boundary problems shows that the solution pair $(\bar{u}, \bar{v})$ and $(\underline{u}, \underline{v})$ satisfy

$$
\begin{cases}-\Delta\left[H_{1}(\bar{u}, \bar{v})\right]+k_{1} H_{1}(\bar{u}, \bar{v})=F_{1}(x, \bar{u}, \bar{v}), & x \in \Omega, \\ -\Delta\left[H_{2}(\bar{u}, \bar{v})\right]+k_{2} H_{2}(\bar{u}, \bar{v})=F_{2}(x, \bar{u}, \bar{v}), & x \in \Omega, \\ -\Delta\left[H_{1}(\underline{u}, \underline{v})\right]+k_{1} H_{1}(\underline{u}, \underline{v})=F_{1}(x, \underline{u}, \underline{v}), & x \in \Omega, \\ -\Delta\left[H_{2}(\underline{u}, \underline{v})\right]+k_{2} H_{2}(\underline{u}, \underline{v})=F_{2}(x, \underline{u}, \underline{v}), & x \in \Omega, \\ \bar{u}(x)=\underline{u}(x)=0, \quad \bar{v}(x)=\underline{v}(x)=0, & x \in \partial \Omega,\end{cases}
$$

which is equivalent to

$$
\begin{cases}-\Delta\left[H_{1}(\bar{u}, \bar{v})\right]=f_{1}(x, \bar{u}, \bar{v}), & x \in \Omega, \\ -\Delta\left[H_{2}(\bar{u}, \bar{v})\right]=f_{2}(x, \bar{u}, \bar{v}), & x \in \Omega, \\ -\Delta\left[H_{1}(\underline{u}, \underline{v})\right]=f_{1}(x, \underline{u}, \underline{v}), & x \in \Omega, \\ -\Delta\left[H_{2}(\underline{u}, \underline{v})\right]=f_{2}(x, \underline{u}, \underline{v}), & x \in \Omega, \\ \bar{u}(x)=\underline{u}(x)=0, \quad \bar{v}(x)=\underline{v}(x)=0, & x \in \partial \Omega .\end{cases}
$$

Therefore $(\bar{u}, \bar{v})$ and $(\underline{u}, \underline{v})$ are its maximal and minimal solutions to problem (1.4), in the sense that if $(u, v)$ is any other solution of $(1.4)$ in $\langle(\hat{u}, \hat{v}),(\tilde{u}, \tilde{v})\rangle$, then they satisfy $(\underline{u}, \underline{v}) \leq$ $(u, v) \leq(\bar{u}, \bar{v})$, as well as the true solutions to problem (1.4).

The above derivations lead to the following theorem.

Theorem 3.3 Let $(\tilde{u}, \tilde{v})$ and $(\hat{u}, \hat{v})$ be a pair of ordered upper and lower solutions of (1.4), then the sequences $\left\{\left(\bar{u}^{(n)}, \bar{v}^{(n)}\right)\right\}$ and $\left\{\left(\underline{u}^{(n)}, \underline{v}^{(n)}\right)\right\}$ provided from (3.10) converge monotonically from above to a maximal solution $(\bar{u}, \bar{v})$ and from below to a minimal solution $(\underline{u}, \underline{v})$ in $S$, respectively, and satisfy the relation

$$
\begin{aligned}
(\hat{u}, \hat{v}) & \leq\left(\underline{u}^{(n)}, \underline{v}^{(n)}\right) \leq\left(\underline{u}^{(n+1)}, \underline{v}^{(n+1)}\right) \leq\left(\bar{u}^{(n+1)}, \bar{v}^{(n+1)}\right) \\
& \leq\left(\bar{u}^{(n)}, \bar{v}^{(n)}\right) \leq(\tilde{u}, \tilde{v}) \quad \text { for } n=1,2, \ldots ;
\end{aligned}
$$

furthermore, if $\bar{u}=\underline{u}$ or $\bar{v}=\underline{v}$, then $(\bar{u}, \bar{v})=(\underline{u}, \underline{v})\left(:=\left(u^{*}, v^{*}\right)\right)$ and $\left(u^{*}, v^{*}\right)$ is the unique solution of (1.4) in $\bar{\Omega}$. 


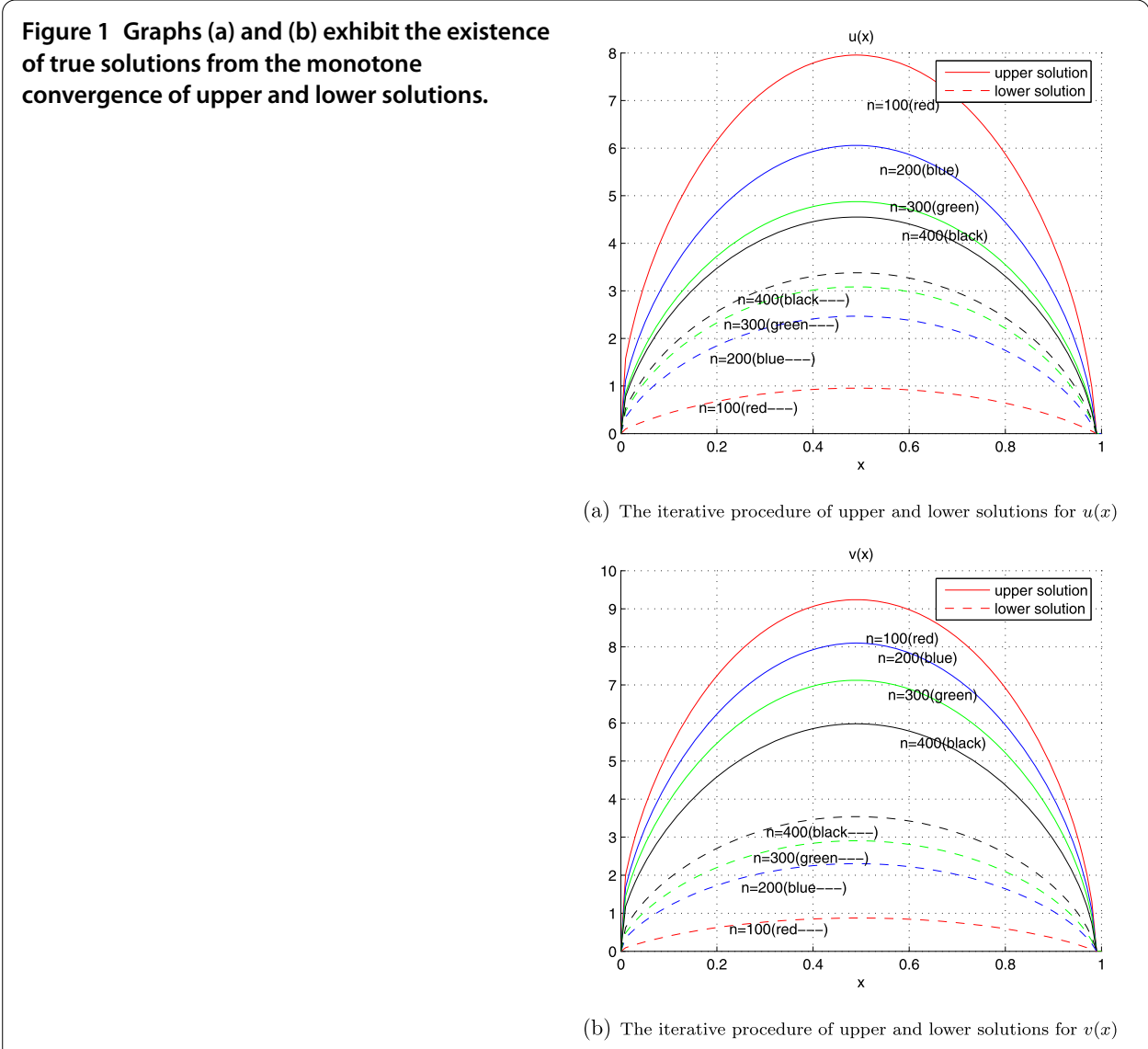

\section{Numerical simulation and discussion}

In this section, we illustrate our theoretical results through numerical simulations. The finite difference method and the Newton-Raphson method are applied in the simulation. To reveal the feather of model (1.3), we now run numerical simulations with the following functions and parameters:

$$
\begin{aligned}
& \Omega=(0,1), \quad d_{1}=0.05, \quad \alpha_{1}=0.03, \quad d_{2}=0.01, \quad \alpha_{2}=0.01, \\
& \gamma_{1}=2.4, \quad \gamma_{2}=2.5, \quad \beta_{1}=\beta_{2}=0.001, \\
& a_{12}(x)=10+0.06 \sin \left(\frac{1}{100} x\right), \quad a_{22}(x)=10+0.2 \sin \left(\frac{1}{100} x\right), \\
& g(u)=\sqrt{u+1}-1, \quad a_{11}(x)=8+0.16 \sin \left(\frac{1}{100} x\right) .
\end{aligned}
$$

With the help of Matlab, we present the iterative procedure by Figure 1. Moreover, we can gain the insight from Figure 1 that the upper solution sequence $\left(\bar{u}^{(m)}, \bar{v}^{(m)}\right)$ is monotone decreasing and the lower solution sequence $\left(\underline{u}^{(m)}, \underline{v}^{(m)}\right)$ is monotone increasing, respectively. Therefore, problem (1.3) admits at least one coexistence solution.

In present paper, we proposed and studied a cross-diffusion fecally-orally epidemic model where we had considered the coexistence solution to problem (1.3) in a spatially heterogeneous environment. Firstly, we introduced the basic reproduction number $R_{0}^{D}$ via the next generation operator and associated linear eigenvalue problem, and we further proved 
that $R_{0}^{D}$ served as a threshold parameter which predicts whether the coexistence exists or not. To be more precise, when all coefficients are constants, we provided an explicit formula for $R_{0}^{D}$. The coexistence of problem (1.3) is investigated by combining the eigenvalue problem and monotone iterative schemes when $R_{0}^{D}>1$, while if $R_{0}^{D}<1$, problem (1.3) has no coexistence solution. We proved that large cross-diffusion will result in non-existence of the coexistence (Theorem 3.2), whereas coexistence is possible if the cross-diffusion coefficients are small (Theorem 3.1). As far as we know, on the propagation of the species, large cross-diffusion will result in more complex dynamic behavior in ecology, for example, large cross-diffusion can destabilize a uniform positive equilibrium which is stable for the ODE system and for the weakly coupled reaction-diffusion system [27]; see also [19, $28]$ and the references therein. Our results show that cross-diffusion has also a significant impact on the coexistence of the epidemic model.

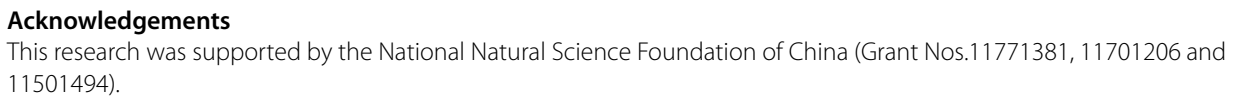

\section{Competing interests}

The authors declare that they have no competing interests.

\section{Authors' contributions}

All authors contributed equally to the writing of this paper. All authors read and approved the final manuscript.

\section{Author details}

'School of Mathematical Science, Huaiyin Normal University, Huaian, 223300, China. ${ }^{2}$ School of Mathematical Science, Yangzhou University, Yangzhou, 225002, China.

\section{Publisher's Note}

Springer Nature remains neutral with regard to jurisdictional claims in published maps and institutional affiliations.

Received: 30 August 2017 Accepted: 8 November 2017 Published online: 28 November 2017

References

1. Capasso, V, Paveri-Fontana, SL: A mathematical model for the 1973 cholera epidemic in the European Mediterranean region. Rev. Epidemiol. Sante Publ. 27, 121-132 (1979) Errata, Ibid. 28, 390 (1980)

2. Capasso, V, Maddalena, L: Convergence to equilibrium states for a reaction-diffusion system modeling the spatial spread of a class of bacterial and viral diseases. J. Math. Biol. 13, 173-184 (1981)

3. Thieme, HR, Zhao, XQ: Asymptotic speeds of spread and traveling waves for integral equations and delayed reaction-diffusion models. J. Differ. Equ. 195, 430 (2003) C470

4. Xu, DS, Zhao, XQ: Asymptotic speed of spread and traveling waves for a nonlocal epidemic model. Discrete Contin. Dyn. Syst., Ser. B 5, 1043-1056 (2005)

5. Wang, YX, Wang, ZC: Entire solutions in a time-delayed and diffusive epidemic model. Appl. Math. Comput. 219, 5033-5041 (2013)

6. Wu, SL: Entire solutions in a bistable reaction-diffusion system modeling man-environment-man epidemics. Nonlinear Anal., Real World Appl. 13, 1991-2005 (2012)

7. Ahn, I, Baek, S, Lin, ZG: The spreading fronts of an infective environment in a man-environment-man epidemic model. Appl. Math. Model. 40, $7082-7101$ (2016)

8. Allen, LS, Bolker, BM, Lou, Y, Nevai, AL: Asymptotic profiles of the steady states for an SIS epidemic reaction-diffusion model. Discrete Contin. Dyn. Syst., Ser. A 21, 1-20 (2008)

9. Lin, ZG, Zhu, HP: Spatial spreading model and dynamics of West Nile virus in birds and mosquitoes with free boundary. J. Math. Biol. 75, 1381-1409 (2017)

10. Lou, Y, Ni, WM: Diffusion vs cross-diffusion: an elliptic approach. J. Differ. Equ. 154, 157-190 (1999)

11. Cai, YL, Wang, WM: Fish-hook bifurcation branch in a spatial heterogeneous epidemic model with cross-diffusion. Nonlinear Anal., Real World Appl. 30, 99-125 (2016)

12. Chen, B, Peng, R: Coexistence states of a strongly coupled prey-predator model. J. Partial Differ. Equ. 18, 154-166 (2005)

13. Gan, W, Lin, Z: Coexistence and asymptotic periodicity in a competitor-competitor-mutualist model. J. Math. Anal. Appl. 337, 1089-1099 (2008)

14. Kuto, K, Yamada, Y: Multiple coexistence states for a prey-predator system with cross-diffusion. J. Differ. Equ. 197, 315-348 (2004)

15. Lou, Y, Tao, YS, Winkler, M: Nonexistence of nonconstant steady-state solutions in a triangular cross-diffusion model. J. Differ. Equ. 262, 5160-5178 (2017) 
16. Pao, CV: Strongly coupled elliptic systems and applications to Lotka-Volterra models with cross-diffusion. Nonlinear Anal. 60, 1197-1217 (2005)

17. Kuto, K: Stability and Hopf bifurcation of steady-state solutions to an SKT model in a spatially heterogeneous environment. Discrete Contin. Dyn. Syst. 24, 489-509 (2009)

18. Wang, YX, Li, WT: Effects of cross-diffusion on the stationary problem of a diffusive competition model with a protection zone. Nonlinear Anal., Real World Appl. 14, 224-245 (2013)

19. Li, SB, Liu, SY, Wu, JH, Dong, YY: Positive solutions for Lotka-Volterra competition system with large cross-diffusion in a spatially heterogeneous environment. Nonlinear Anal., Real World Appl. 36, 1-19 (2017)

20. Xu, DS, Zhao, XQ: Bistable waves in an epidemic model. J. Dyn. Differ. Equ. 16, 679-707 (2004)

21. Diekmann, O, Heesterbeek, JAP, Metz, JAJ: On the definition and the computation of the basic reproduction ratio $R_{0}$ in models for infectious diseases in heterogeneous populations. J. Math. Biol. 28, 365-382 (1990)

22. Wang, WD, Zhao, XQ: Basic reproduction numbers for reaction-diffusion epidemic models. SIAM J. Appl. Dyn. Syst. 11, 1652-1673 (2012)

23. Peng, R, Zhao, XQ: A reaction-diffusion SIS epidemic model in a time-periodic environment. Nonlinearity 25 , 1451-1471 (2012)

24. Thieme, HR: Spectral bound and reproduction number for infinite dimensional population structure and time heterogeneity. SIAM J. Appl. Math. 70, 188-211 (2009)

25. van den Driessche, $P$, Watmough, J: Reproduction numbers and sub-threshold endemic equilibria for compartmental models of disease transmission. Math. Biosci. 180, $29-48$ (2002)

26. Kim, KI, Lin, ZG: Coexistence of three species in a strongly coupled elliptic system. Nonlinear Anal. 55, 313-333 (2003)

27. Fu, SM, Zhang, L: Instability induced by cross-diffusion in a predator-prey model with sex structure. J. Appl. Math. 2012, Article ID 240432 (2012)

28. Kuto, K, Yamada, Y: Positive solutions for Lotka-Volterra competition systems with large cross-diffusion. Appl. Anal. 89 , 1037-1066 (2010)

\section{Submit your manuscript to a SpringerOpen ${ }^{\circ}$ journal and benefit from:}

- Convenient online submission

- Rigorous peer review

- Open access: articles freely available online

- High visibility within the field

- Retaining the copyright to your article

Submit your next manuscript at $\gg$ springeropen.com 